\title{
Labyrinthe
}

34 | 2010 (1)

Comment peut-on être systématique?

\section{Quel système pour le jeu théâtral ?}

\section{Laurence Marie}

\section{(2) OpenEdition}

\section{Journals}

Édition électronique

URL : http://journals.openedition.org/labyrinthe/4058

DOI : $10.4000 /$ labyrinthe.4058

ISSN : 1950-6031

Éditeur

Hermann

Édition imprimée

Date de publication : 1 février 2010

Pagination : 81-91

ISBN : 978-2-7056-6984-3

\section{Référence électronique}

Laurence Marie, «Quel système pour le jeu théâtral ? », Labyrinthe [En ligne], 34 | 2010 (1), mis en ligne le 01 février 2012, consulté le 21 avril 2019. URL : http://journals.openedition.org/labyrinthe/4058 ;

DOI : $10.4000 /$ labyrinthe.4058 


\title{
Quel système pour le jeu théâtral?
}

\author{
Laurence Marie \\ lau.marie@gmail.com
}

Les premiers ouvrages exclusivement consacrés au jeu du comédien de théâtre ne paraissent pas avant le milieu du XVIII ${ }^{\mathrm{e}}$ siècle. Dans les années 1740-1770, plusieurs auteurs s'interrogent explicitement sur la manière d'établir et d'organiser les principes d'une pratique abordée de manière secondaire dans les traités d'art oratoire, où l'acteur est envisagé comme un double médiocre de l'orateur en chaire, à la tribune ou au barreau.

À un moment où les Encyclopédistes se lancent dans un travail de systématisation des connaissances et où la notion même de système tend à changer de sens et de portée, analyser spécifiquement le cas du jeu théâtral permet de réfléchir sur la façon d'organiser un savoir qui n'a pas été théorisé en tant que tel jusque-là.

Les auteurs de traités de jeu s'attachent à identifier les matières propres à la pratique du comédien et à les mettre en ordre. S'agit-il pour autant d'une mise en système, au sens donné par l'Encyclopédie («le tout et l'ensemble d'une théorie dont les différentes parties sont liées entre elles, se suivent et dépendent les unes des autres $\left.{ }^{1} \gg\right)$ ? Quels sont les enjeux esthétiques de ces tentatives d'établir un organon de règles pour une pratique considérée jusqu'alors comme bâtarde et dépourvue de légitimité artistique? Enfin, plus largement, en quoi les premiers ouvrages portant sur le jeu théâtral contribuent-ils à nuancer une approche critique rationaliste de l'entreprise théorique qui est celle des Lumières?

Les premiers auteurs de traités de jeu cherchent chacun à leur manière à souligner l'aspect novateur de leur démarche analytique. Pour ne prendre qu'un exemple, Rémond de Sainte-Albine, auteur du Comédien, premier traité de jeu français, publié en 1747 , écrit dès la préface qu'il veut élaborer la « théorie d'un Art de goût » et « faire le premier cet essai

1. Denis Diderot et Jean le Rond d'Alembert, Encyclopédie, ou Dictionnaire raisonné des sciences, des arts et des métiers, Neuchastel, Samuel Faulche, t. Xv, 1765, p. 778. 
sur un Art qui renferme autant de parties que l'Art du Comédien, et sur les principes duquel on est si peu d'accord $»^{2}$.

Les théoriciens justifient leur approche en affirmant vouloir contrer l'opinion selon laquelle jouer résulte d'une improvisation spontanée et ne s'apprend pas. C'est précisément ce type de préjugé que moque Henry Fielding dans Tom Jones, en mettant en scène le valet Partridge. Celui-ci emporté par l'illusion suscitée par la représentation de Hamlet, dénie tout talent au grand comédien David Garrick ${ }^{3}$. Le jeu suit en somme un itinéraire théorique similaire à celui emprunté par l'art du poète au siècle précédent: plutôt que d'être le simple fruit d'un enthousiasme inspiré, il est désormais appréhendé comme un art exigeant un travail assidu ${ }^{4}$.

Les auteurs de traités s'engagent dans une entreprise de rationalisation des éléments du spectacle et de remise en cause des fausses évidences. Sainte-Albine souhaite ainsi « soumettre au raisonnement et à l'analyse diverses vérités, qui semblent n'être que du ressort du sentiment ${ }^{5}$ ». L'enjeu de cette intellectualisation du jeu est crucial: tel est en effet le principal critère qui distingue la «profession » et le « métier $^{6} »$, les arts libéraux, arts de «l'esprit», et les arts mécaniques, arts de la « main ${ }^{7} »$. Établir des règles permet de différencier la nature et le naturel, de faire du comédien un imitateur à part entière, au sens mélioratif du terme, et d'accréditer l'idée qu'il puisse exister un savoir propre au jeu. La théorie du jeu prend dès lors toute sa légitimité, ce qui lui ouvre la voie de l'autonomie par rapport à la rhétorique oratoire, son ascendance noble qui la condamnait à un statut subalterne.

Plusieurs théoriciens estiment ainsi que le jeu doit être doté de règles pour pouvoir être considéré comme un art. Le comédien allemand Ekhof insiste par exemple en 1753 sur l'opportunité d'élaborer une " grammaire du jeu » (Grammatik der Schauspielkunst) qui remonte aux «fondements »(Ursachen) et hausse le comédien au rang d' «artiste libéral »

2. Pierre Rémond de Sainte-Albine, Le Comédien, Paris, Desaint et Saillant, 1747, p. 5-6.

3. Voir Henry Fielding, Tom Jones, livre Xvı, chap. v, dans Romans, éd. Francis Ledoux, Paris, Gallimard, Pléiade, 1964, p. 1391 et Tom Jones, éd. Sheridan Baker, New York/Londres, Norton, 1973, p. 659.

4. Voir Paul Bénichou, Le Sacre de l'écrivain. Le Temps des prophètes, dans Romantismes français,

t. I, Paris, Gallimard, 1996 [1973], chap. I.

5. Pierre Rémond de Sainte-Albine, op. cit., p. 5.

6. Voir notamment William Cooke, The Elements of dramatic criticism, Londres, Kearsly, 1775, chap. XVIII, p. $178 s q$.

7. Denis Diderot, «Art », dans Encyclopédie, éd. citée, t. I, 1751, p. 715. 


\section{Quel système pour le jeu théâtral?}

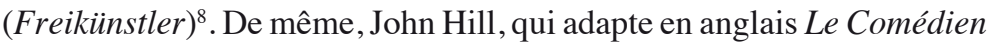
de Sainte-Albine, déclare en 1755 que « le jeu est une science, et doit être étudié comme une science » (Playing is a science, and is to be studied as a science $e^{9}$ ). Hausser le jeu au statut de « connaissance claire et certaine [...], fondée ou sur des principes évidents par eux-mêmes, ou sur des démonstrations ${ }^{10}{ }^{\prime}$, c'est clairement l'élever au rang d'art, puisque «l'art » et la « science » sont, à la suite du XVII ${ }^{\mathrm{e}}$ siècle, encore synonymes au XVIII ${ }^{\mathrm{e}}$ siècle, selon une approche cognitive fortement normative ${ }^{11}$.

Donner des règles à un jeu théâtral par essence transitoire et éphémère, c'est aussi l'aider à résister au passage du temps et favoriser sa transmission et son progrès ${ }^{12}$; c'est lui conférer, au moins en partie, la pérennité qui lui manque pour figurer en haut de la hiérarchie des beaux-arts, selon le critère énoncé par Watelet à l'ouverture de son Encyclopédie méthodique (1788) : pour lui en effet, les « arts ou langages dont les productions sont fixes et durables » (arts de la sculpture, de l'architecture et de la peinture) sont supérieurs à ceux « dont les productions sont transitoires ou instantanées ${ }^{13} »$ (arts de la pantomime, de la parole et de la musique).

Faire reconnaître que le jeu est guidé par des principes spécifiques conduit dès lors à modifier un système des arts gouverné par une hiérarchie qui perdure depuis le siècle précédent. Le jeu est redéfini en relation avec les autres arts, et en particulier la peinture et la danse, qui reposent sur l'expression visuelle des passions. Si la plupart des théoriciens comparent

8. Conrad Ekhof, Journal der Academie der Schönemannischen Gesellschaft, séance du 30 juin 1753, cité par Wolfgang F. Bender, « Vom "tollen Handwerk" zur Kunstübung. Zur "Grammatik" der Schauspielkunst im 18. Jahrhundert », dans Wolfgang F. Bender (dir.), Schauspielkunst im 18. Jahrhundert, Stuttgart, Steiner, 1992, p. 16.

9. John Hill, The Actor: A Treatise on the Art of Playing [L'Acteur: traité sur l'art du jeu], Londres, R. Griffiths, 1755 [1750], p. 12.

10. « Science », dans Encyclopédie, op. cit., t. XIV, 1765, p. 787.

11. Dans la continuité de Furetière («Art », dans Antoine Furetière, Dictionnaire universel, 1791, t. I, non paginé), l'auteur de l'article «Science» (Encyclopédie, op. cit., t. XIV, 1765, p. 787-788) définit « l'art » comme l'une des quatre branches de la science. L'Encyclopédie distingue les arts et les sciences, tout en montrant l'affinité qu'ils entretiennent: l'art y renvoie à la « collection et la disposition technique des règles selon lesquelles [un objet] s'exécute », la science désignant un objet qui se contemple plutôt qu'il ne s'exécute («Art », dans Encyclopédie, opc. cit., t. I, 1751, p. 715).

12. Voir Jean-Nicolas Servandoni, dit d'Hannetaire, Observations sur l'art du comédien, Aux dépends d'une société typographique, 1774 [1772], p. 26-29 et Claude-Joseph Dorat, La Déclamation théâtrale, poème didactique en trois chants, Paris, Sébastien Jorry, 1766, p. 7.

13. Claude-Henri Watelet, Encyclopédie méthodique, ou par ordre de matières; par une société de gens de lettres, de savans et d'artistes, Paris, Panckoucke et Liège, Plomteux, t. I, 1788, « Notions préliminaires: la peinture est un art libéral », p. III. Le jeu étant un composé des arts de la pantomime et de la parole, il participe de la seconde catégorie définie par Watelet. 
le travail du comédien à celui du peintre, en 1759, l'Anglais Wilkes va plus loin : réactivant le topos de la concurrence entre les arts, il place le jeu au faîte de la hiérarchie, soutenant avec audace que «LE JEU est le plus parfait de tous les arts imitatifs, car il est composé de tout ce qui est beau en Poésie, en Peinture et en Musique ${ }^{14} »$. Cette déclaration reste marginale dans le corpus des traités de jeu de la seconde moitié du XVIII ${ }^{e}$ siècle. Mais elle n'en est pas moins significative d'une volonté de faire du jeu une sorte d'archi-art, réunissant les qualités de tous les autres, pour l'intégrer de force au sein d'un système des arts qui ne le mentionne pas en tant que tel. Le succès officiel de la campagne de revalorisation menée par les auteurs reste mitigé. Dans le «Discours préliminaire » de 1751, D'Alembert fait entrer dans les «beaux-arts » « la Peinture, la Sculpture, l'Architecture, la Poésie, et leurs différentes divisions »; sans évoquer ni les « arts de la pantomime », ni ceux de la "parole », qui composent désormais le jeu à parts égales. Watelet intégrera ces derniers dans son système des arts en 1788 , tout en précisant qu'ils figurent au bas de l'échelle ${ }^{15}$.

Qui sont, dans cette perspective de légitimation théorique du jeu, les auteurs autorisés à écrire sur cette matière nouvelle ? De quelle méthode usent-ils pour identifier les matières propres au jeu et les classifier?

Plutôt que de se contenter de remanier les principes institués par l'art de l'orateur, les auteurs se livrent à une véritable refondation, en justifiant la méthode par laquelle ils ont élaboré leur assemblage de règles. Plutôt que de s'inspirer des codes de l'art oratoire en les envisageant comme des principes posés a priori et valant universellement, ils affichent leur volonté de remonter aux origines, en adoptant la méthode empirique défendue par Condillac dans le Traité des systèmes ${ }^{16}$ : selon eux, c'est en observant directement la nature que l'on peut mettre au

14. Wilkes, A General View of the Stage, Londres, Coote, 1759, p. 82 : «ACTING is the most perfect of all the imitative Arts, as being made up of all that is beautiful in Poetry, Painting, and Music ».

15. Claude-Henri Watelet, op. cit., p. III.

16. Étienne Bonnot, abbé de Condillac, Traité des systèmes, 1749, Paris, Fayard, 1991, chap. XVII: «De l'usage des systèmes dans les arts »,p. 261-264. Le texte a été écrit avant 1780. Tous les articles de ce dossier soulignent comment la nouvelle démarche empirique est le principal levier qui remet en cause l'approche classique du système. Voir en particulier le texte de Déborah Cohen, qui montre l'implication d'un tel renversement de perspective sur la théorisation du droit ayant, comme le jeu théâtral, des implications directement pratiques. 


\section{Quel système pour le jeu théâtral?}

jour les règles qu'elle contient et qui gouvernent le jeu ${ }^{17}$. Les auteurs affirment ainsi n'avoir rien inventé, mais avoir simplement rassemblé des principes présents dans la nature ${ }^{18}$.

Cette manière d'appréhender la définition des règles ouvre aux spectateurs de théâtre la possibilité de théoriser ${ }^{19}$. Un débat émerge alors: qui, des spectateurs ou des comédiens, est le plus apte et le plus légitime à donner au jeu l'arsenal de règles qui lui fait défaut? Le polygraphe Sainte-Albine ${ }^{20}$ affirme à l'ouverture du Comédien que ce sont sans conteste les premiers puisque «sur les Arts, qui puisent leurs principes dans la nature et dans la raison, tout homme sensible et raisonnable a droit de hasarder ses conjectures ». À ses yeux, le spectateur assidu est plus apte à réfléchir sur le jeu, n'étant pas juge et partie, à la différence du praticien de théâtre ${ }^{21}$. À l'inverse, de plus en plus nombreux sont les théoriciens à fonder l'objectivité du jugement et la capacité à théoriser sur une expérience de praticien du théâtre. Le comédien et directeur de théâtre d'Hannetaire, opposant le «bon Peintre de profession » au « simple amateur », se targue d'avoir tiré ses prescriptions de trente ans de pratique du jeu et de l'enseignement ${ }^{22}$.

Mais qu'ils soient spectateurs ou acteurs, les premiers auteurs de jeu se rejoignent sur un élément: prenant pour point de départ de leur tentative de systématisation une expérience sensorielle du spectacle, ils remontent de l'effet qu'il produit à la manière de le produire. De manière novatrice, ils participent donc à la redéfinition de la production artistique comme une relation esthétique ${ }^{23}$ entre un sujet créateur et un sujet récepteur, plutôt que comme soumission aux règles d'une poétique. En ce sens, les premiers écrits

17. De fait, l'attrait pour l'empirisme ne met pas en cause l'idée selon laquelle les phénomènes naturels sont organisés par des lois nécessaires et non contingentes (voir André Charrak, Contingence et nécessité des lois de la nature au XVIII siècle. La philosophie seconde des Lumières, Paris, Vrin, 2006).

18. Voir notamment Aaron Hill, The Art of Acting, Londres, Osborn, 1746, p. VII.

19. De même, au siècle précédent, des « amateurs » comme Roger de Piles, membres de l'Académie Royale de Peinture et de Sculpture fondée en 1648, théorisent la peinture sans être eux-mêmes peintres. 20. Membre de l'Académie royale des Sciences et Belles-Lettres de Berlin, censeur du roi, Sainte-Albine publie un Mémoire sur le laminage du plomb en 1731 et un Abrégé de l'Histoire de De Thou en 1759. Il collabore à L'Europe savante, à la Gazette de France et au Mercure de France, qu'il dirige quelque temps.

21. Pierre Rémond de Sainte-Albine, op. cit., 1747, p. 8.

22. Jean-Nicolas Servandoni, dit D'Hannetaire, op. cit., 1774, p. 13 et p. 235.

23. Nous envisagerons ici ce mot au sens que lui donne son créateur, l'Allemand Alexandre Baumgarten, au milieu du XVIII ${ }^{\mathrm{e}}$ siècle : la science de la connaissance sensible ou « science de l'art » (Kunstwissenschaft) qui prend pour objet l'émotion produite par le beau. Sur la naissance de cette conception, voir Baldine Saint-Girons, «L'esthétique: problèmes de définition », dans Serge Trottein (dir.), L'Esthétique naîtelle au XVIII siècle?, Paris, PUF, 2000, p. 81-118. 


\section{Labyrinthe, $n^{\circ} 34$}

portant sur le comédien s'inscrivent dans une évolution d'ensemble de la pensée de l'art au XVIII ${ }^{\mathrm{e}}$ siècle, à laquelle ils contribuent directement.

Ce type de démarche, fondée sur une analyse de l'effet produit par la représentation, conduit à revaloriser la dimension visuelle du spectacle et à donner un point de départ nouveau à la théorisation. Le visuel suscitait la méfiance des classiques, pour lesquels il excitait de manière pernicieuse les sens du spectateur au lieu de toucher son seul esprit ${ }^{24}$. C'est pourquoi, au $\mathrm{XVII}^{\mathrm{e}}$ siècle et encore au XVIII ${ }^{\mathrm{e}}$ siècle, les auteurs de traités d'éloquence s'attachaient avant tout à la manière de dire le texte: ils accordaient la préséance à la déclamation, le geste ne se voyant accorder qu'une place subalterne, reléguée à la fin des ouvrages ${ }^{25}$. C'est donc à un véritable renversement de perspective que se livrent les théoriciens du jeu qui, sous l'influence du sensualisme, choisissent, comme Sainte-Albine, de consacrer leur premier chapitre, c'est-à-dire la pierre d'angle de leur raisonnement, au sentiment éprouvé par le comédien ou, comme Antoine François Riccoboni, à la gestuelle ${ }^{26}$.

À partir de ce nouveau point de départ visuel, sensoriel et naturel, les auteurs s'écartent de la composition traditionnelle des traités d'art oratoire: ils ne déroulent pas une suite de préceptes concernant la voix et la gestuelle, envisagés séparément. Ils n'établissent pas non plus un catalogue des accents et des gestes prescrits pour chaque passion ${ }^{27}$, démarche traditionnelle que continuent d'adopter certains théoriciens britanniques $^{28}$. Au contraire, ils optent pour une approche plus globale qui échappe à la simple taxinomie. Sainte-Albine s'attache ainsi d'abord aux qualités innées demandées au comédien, suivant en cela Cicéron ${ }^{29}$.

24. Sur cette question, voir Angelica Goodden, Action and Persuasion, Dramatic Performance in Eighteenth-Century France, Oxford, Clarendon Press, 1986, p. 20-21 et p. 37.

25. Même le premier grand ouvrage consacré à l'éloquence du corps, le Traité de l'action de l'orateur ou de la prononciation et du geste, publié par Michel Le Faucheur en 1657, commence par aborder la prononciation et ne s'attaque au geste qu'aux chapitres XII et XIII, suivis par le chapitre de conclusion. 26. Antoine François Riccoboni, L'Art du théâtre, Paris, Simon et Giffart fils, 1750, p. 4: « je vais commencer par vous parler du geste, et cela vous paraîtra peut-être bizarre ».

27. La Méthode pour bien prononcer un discours, et pour le bien animer de René Bary est composée d'une suite de chapitres commençant invariablement par « De l'accent », puis par « Du geste » : on trouve ainsi «De l'accent de l'Amour », « De l'accent de la Haine », « Du geste de la tendresse », « Du geste du triomphe », etc. Le texte a été réédité par Sabine Chaouche dans Sept Traités sur le jeu du comédien et autres textes, De l'action oratoire à l'art dramatique (1657-1750), Paris, Champion, 2001.

28. C'est le cas d'Aaron Hill et de Samuel Foote (A Treatise on the passions, so far as they regard the stage, Londres, Corbet, 1747), qui adaptent au théâtre les symptômes passionnels répertoriés et décrits par les orateurs, les philosophes rationalistes et le peintre Le Brun.

29. Cicéron, De l'orateur, texte établi et traduit par Edmond Courbaud, Paris, Belles Lettres, 1985, livre I. 


\section{Quel système pour le jeu théâtral?}

Puis il s'intéresse, dans la seconde partie, aux différents éléments qui alimentent la « vérité de la représentation ». Son ouvrage, subdivisé en de nombreuses sections et chapitres, hiérarchise et agence les matières dans une visée totalisante qui les fait se succéder selon une logique démonstrative où chaque partie du jeu dépend des autres. La représentation théâtrale, ainsi revalorisée par rapport au texte, est pour la première fois considérée comme un réseau organique de signes, un système visuel où tout fait sens pour susciter l'illusion.

L'ambition qui préside à l'ouvrage d'Antoine François Riccoboni, beaucoup plus synthétique que l'imposante somme de Sainte-Albine, paraît elle aussi faire écho à la définition du système donnée dans l'Encyclopédie. Selon lui en effet, « tout l'art du théâtre se réduit à un très petit nombre de principes $^{30} »$. Bien que les chapitres («Le Geste», «La Voix », « La Déclamation », «L'intelligence », «L'Expression », etc.) paraissent au premier abord distribués sans ordonnancement particulier, l'auteur explique en conclusion que « c'est en suivant l'ordre que je viens de prescrire dans l'étude du Théâtre, que l'on parvient à se rendre capable de tout exprimer en quelque situation que ce puisse être ${ }^{31} »$.

Mais si la plupart des auteurs des années 1740-1770 affirment s'adresser aux comédiens pour les former, il ne semble pas que les premiers traités français aient exercé une influence sur la pratique scénique. Certes, le comédien Riccoboni destine L'Art du théâtre à "Madame*** », qu'il présente comme une spectatrice aimant à jouer la comédie sur des théâtres privés. De même, Sainte-Albine affirme que

son intention a été, en attendant qu'il paraisse sur l'Art du Comédien un Traité tel qu'on aurait droit de désirer, d'aider les personnes qui veulent embrasser cette Profession, à connaître si elles sont propres au Théâtre, et à découvrir quelques-uns des moyens, par lesquels elles peuvent espérer de s'y faire applaudir.

Il affirme s'être bien gardé de déduire d'une hypothèse quelques réflexions fines et générales, et $[\ldots]$ créer un ingénieux système, qui aurait pu servir de base aux règles de l'Art, mais dans lequel les Artistes ne les auraient pas aperçues ${ }^{32}$.

30. Antoine François Riccoboni, op. cit., p. 101.

31. Ibid. Nous soulignons.

32. Pierre Rémond de Sainte-Albine, op. cit., p. 10-11. 


\section{Labyrinthe, $n^{\circ} 34$}

Sainte-Albine refuse le système comme une construction abstraite inutile aux hommes de l'art. Cependant, son ouvrage s'apparente moins à un manuel pratique de jeu qu'à une poétique, dans la lignée de celles établies au siècle précédent pour la poésie, la peinture ou l'art oratoire. Au-delà des précautions d'usage, Sainte-Albine ne doute pas véritablement de la capacité de la raison à rendre compte des arcanes du jeu théâtral ${ }^{33}$. Ironie du sort: alors même que Sainte-Albine se défend de tout esprit de système, la critique principale qui lui sera adressée, c'est justement d'avoir voulu constituer un système philosophique clos, sans prise sur la réalité du spectacle. L'Allemand Lessing décrit ainsi son ouvrage en 1754 comme une « belle métaphysique du jeu du comédien » (eine schöne Metaphysik von der Kunst des Schauspielers ${ }^{34}$ ), qui n'a d'enjeu autre que théorique.

De fait, les premiers traités de jeu, qu'ils soient écrits par des spectateurs ou par des comédiens, réunissent un ensemble de règles qui sont « plus destinées à rendre raison des effets qu'à apprendre à les produire ${ }^{35}$ », selon la définition des règles des beaux-arts donnée par Condillac dans son Traité des systèmes (1749). Les principes normatifs donnés par les auteurs répondent à une stratégie de légitimation du jeu plus qu'à une visée véritablement pratique. Ils s'adressent finalement moins aux acteurs qu'aux spectateurs, auxquels ils fournissent des critères de jugement, en relation avec une approche plus normative du goût influencée par l'Angleterre, et par Hume en particulier ${ }^{36}$.

Toutefois, quelques théoriciens critiquent toute velléité de réduire le jeu à un corps de préceptes, en préférant à ceux-ci l'étude directe de la nature. Ainsi Diderot affirme-t-il dans le Paradoxe sur le comédien que la lecture des préceptes de Sticotti, adaptateur de seconde main de Sainte-Albine ${ }^{37}$, n'est d'aucune utilité au comédien:

\footnotetext{
33. En ce sens, et aussi parce que le théoricien réfléchit à la façon dont le comédien peut contrôler l'expression du sentiment naturel en usant de son « esprit », l'entreprise de Sainte-Albine est guidée par cette « raison totalitaire » dont parlent Marion Chottin et Arnault Skornicki pour montrer que les Lumières ne s'y réduisent pas.

34. Gotthold Ephraïm Lessing, «Auszug aus dem "Schauspieler" des Herrn Remond von Sainte Albine », dans Lessings Sämtliche Werke in zwanzig Bänden, éd. Hugo Görin, t. vII: «Theatralische Bibliothek I, 1754-1758 », Stuttgart/Berlin, Gotta’sche Buchhandlung Nachfolger, 1893, p. 123.

35. Condillac, op. cit., p. 247.

36. Voir Jean-François Groulier, « Goût », dans Barbara Cassin (dir.), Vocabulaire européen des philosophies, Paris, Seuil/Le Robert, 2004, p. 519.

37. Michel Sticotti traduit librement la seconde édition de The Actor de John Hill (1755), lui-même traducteur-adaptateur du Comédien de Sainte-Albine pour le public anglais.
} 


\section{Quel système pour le jeu théâtral?}

c'est à la nature à donner les qualités de la personne, la figure, la voix, le jugement, la finesse. C'est à l'étude des grands modèles, à la connaissance du cœur humain, à l'usage du monde, au travail assidu, à l'expérience, et à l'habitude du théâtre, à perfectionner le don de nature ${ }^{38}$.

Le philosophe adresse indirectement à Sainte-Albine une critique du même ordre que celle formulée par Lessing. Il est du reste symptomatique que, pour analyser le jeu, il préfère au traité qui hiérarchise et enchaîne les matières la forme déliée de l'essai et du dialogue contradictoire, qui laisse le champ libre à l'interprétation.

De fait, la critique de l'imitation des règles qui se généralise dans les années 1740-1750 conduit progressivement à valoriser ce qui échappe à la systématisation: le génie. Dans le « Discours préliminaire » ouvrant le premier tome de l'Encyclopédie (1751), D'Alembert affirme ainsi que

la pratique des beaux Arts consiste principalement dans une invention qui ne prend guère ses lois que du génie; les règles qu'on a écrites sur ces Arts n'en sont proprement que la partie mécanique; elles produisent à peu près l'effet du Télescope, elles n'aident que ceux qui voient ${ }^{39}$.

Le philosophe n'inclut pas explicitement le jeu dans les beauxarts. Toutefois, ce type d'analyse informe largement plusieurs écrits consacrés au comédien, qui défendent l'idée selon laquelle les acteurs de génie gagnent certes à étudier les règles pour se les approprier, mais les dépassent pour ouvrir des voies nouvelles. Ainsi, pour le théoricien de la danse pantomime Jean-Georges Noverre, alors que le fait d'imiter toujours selon le même goût " dégénère en habitude », "l'opération du génie, de cet esprit créateur » « enfant[e] tant d'objets divers ${ }^{40} »$. De même, selon le comédien anglais Garrick, le génie convertit le public à son style

\footnotetext{
38. Denis Diderot, Paradoxe sur le comédien, dans Euvres, éd. Laurent Versini, Paris, Robert Laffont, t. Iv p. 1378.

39. Jean le Rond, dit D'Alembert, « Discours préliminaire », Encyclopédie, op. cit., t. I, 1751, p. XIII. D'Alembert hésite entre les dénominations « beaux-arts », «poésie » et « peinture » pour désigner les arts situés au sommet de la hiérarchie. Il propose de « renfermer [les Beaux-Arts] sous le titre général de Peinture, puisque tous les Beaux-Arts se réduisent à peindre, et ne diffèrent que par les moyens qu'ils emploient » et de « les rapporter tous à la Poésie, en prenant ce mot dans sa signification naturelle, qui n'est autre chose qu'invention ou création » (ibid., p. XVII).

40. Jean-Georges Noverre, Lettres sur la danse et les arts imitateurs, 1760-1807, Paris, Lieutier, 1952, p. 183-184. Cette lettre a été publiée dès l'édition de 1760.
} 
particulier, plutôt qu'il ne se plie au goût de celui-ci ${ }^{41}$. L'originalité est valorisée de manière inédite, en ce qu'elle ouvre des routes nouvelles à la représentation théâtrale ${ }^{42}$. Elle a en ce sens une dimension exemplaire qui invite à en faire le point de départ d'un nouvel agencement de règles. Mais c'est sans compter avec le fait qu'elle résiste à sa transcription en mots. Le Français Dorat le remarque: pour lui, les acteurs inspirés par un élan naturel qui les fait sortir du cadre d'une imitation réglée suscitent l'aphasie momentanée des critiques. Les « écarts » montrés par ces génies arrachés à eux-mêmes par l'enthousiasme, tels Lekain et $\mathrm{M}^{\text {lle }}$ Dumesnil, « enlève[nt] au Spectateur le temps de l'examen, et au Critique le froid compas de l'analyse ${ }^{43} \gg$. Faire entrer dans un système le génie ainsi défini, c'est le déposséder de son essence.

Pour autant, aux yeux de ces théoriciens, si le génie introduit du jeu dans les rouages et résiste à sa mise en système, ce n'est pas parce qu'il crée quelque chose qui serait hors nature. Il met au jour de nouvelles règles déjà présentes dans la nature et restées inaperçues, dans la continuité d'une conception du génie héritée du siècle précédent. C'est seulement à partir de la fin du XVIII ${ }^{\mathrm{e}}$ siècle que les théoriciens du jeu valoriseront le génie violemment irrégulier défini par Saint-Lambert et Diderot à l'article «Génie » de l'Encyclopédie ${ }^{44}$. Ils seront influencés par l'idée novatrice de l'Anglais Shaftesbury, selon lequel le génie n'imite pas, mais produit ${ }^{45}$.

La conception nouvelle du génie qui se développe à l'aube du romantisme met à mal «l' "esprit de système", consistant à tout ramener à des principes abstraits », tel que le définit André Charrak dans l'entretien. Mais elle montre aussi les limites de «l' "esprit systématique" » : ce dernier, qui, selon A. Charrak, « révèle des zones ordonnées sous des principes

\footnotetext{
41. M. Garrick to M. Powell, Paris, 12 décembre 1764, dans The Private Correspondence with the most celebrated persons of his time, Londres, H. Colburn et R. Bentley, 1832, p. 177.

42. Voir Roland Mortier, L'Originalité: une nouvelle catégorie esthétique au siècle des Lumières, Genève, Droz, 1982.

43. Claude Joseph Dorat, op. cit., p. 20-21.

44. Voir Saint Lambert, « Génie », dans L'Encyclopédie, éd. citée,t. xv, p. 37 (Diderot aurait contribué à l'article).

45. Voir Anton Ashley Cooper Shaftesbury, A Letter concerning Enthusiasm to my Lord *****, 1707, Londres, J. Morphew, 1708, p. 81. Voir aussi Alain Pons, « Génie », dans B. Cassin (dir.), Vocabulaire, éd. citée, p. 498.
} 


\section{Quel système pour le jeu théâtral?}

lisibles, mais qui ne prétend pas expliciter leur cohérence globale ${ }^{46}{ }$, implique en effet que le système rende compte d'un ordre naturel existant, dont certaines parties n'ont pas encore pu être appréhendées par la raison.

Pour autant, reconnaître l'existence d'un génie qui ne met pas seulement au jour ce qui existe, mais qui crée du nouveau, ne disqualifie pas toute tentative de rassembler un ensemble de règles mécaniques qui ne soit pas un système totalisant, mais qui ait une valeur propédeutique pour l'apprenti comédien, génie en puissance.

C'est ici que, dans notre perspective, la notion d' « agencement » proposée par Diogo Sardinha prend toute sa valeur. Elle permet de sortir d'un raisonnement binaire fondé sur les couples antinomiques raison/ génie, nature/hors nature, système/hors système. En tant que « relation dynamique », qui «ne concerne pas ce qui est présent-étant-là, mais plutôt ce qui est actuel ou en acte », l'agencement est en prise directe avec l'essence même du théâtre, qui par nature résiste à toute systématisation: la mise en action (au sens étymologique d'actio : le geste qui soutient la parole) d'un texte qui est toujours en acte, toujours prêt à s'actualiser à travers le corps d'un acteur, de manière transitoire et singulière.

46. Voir l'entretien avec André Charrak, p. 30. 